\title{
Adsorption of Methylamine at the Surface of Ice. A Grand Canonical Monte Carlo Simulation Study
}

$$
\begin{gathered}
\text { Veronika Szentirmai }^{\mathrm{a}} \text {, Milán Szőri }{ }^{\mathrm{a}, *}, \text { Sylvain Picaud }{ }^{\mathrm{b}} \text {, Pál } \\
\text { Jedlovszky }{ }^{\mathrm{c}, \mathrm{d}, *}
\end{gathered}
$$

${ }^{a}$ Department of Chemical Informatics, Faculty of Education, University of Szeged, Boldogasszony sgt. 6. H-6725 Szeged, Hungary

${ }^{\mathrm{b}}$ Institut UTINAM (CNRS UMR 6213), Université Bourgogne Franche-Comté, 16 route de Gray, F-25030 Besançon, France

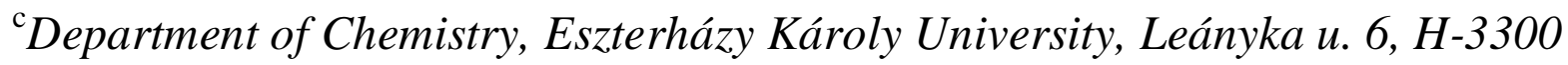
Eger, Hungary

${ }^{\mathrm{d}}$ MTA-BME Research Group of Technical Analytical Chemistry, Szt. Gellért tér 4, H-1111 Budapest, Hungary

Running title: Adsorption of methylamine on ice

*E-mail: milan@jgypk.u-szeged.hu (M.Sz.), pali@chem.elte.hu (P.J.); phone: +36-70-3357820 (M.Sz.), +36-20-9881527 (P.J.) 


\section{Abstract:}

A series of 41 Monte Carlo simulations are performed in the grand canonical ensemble at $200 \mathrm{~K}$ to determine the adsorption isotherm and study in detail the adsorption of methylamine at the surface of $I_{h}$ ice. The adsorption isotherm exhibits a plateau, corresponding to the saturated adsorption monolayer, in a broad range of chemical potentials and pressures. However, even this part of the adsorption isotherm deviates noticeably from the Langmuir shape. Shortly before condensation of methylamine occurs outer molecular layers also start building up. The remarkable stability of the adsorption monolayer is caused by the interplay of hydrogen bonding interaction between the adsorbed methylamine and surface water molecules and dipolar interaction between neighboring adsorbed methylamines. As a consequence, the adsorbed methylamine molecules exhibit a rich orientational distribution relative to the ice surface and the adsorption is accompanied by rather large energy variations. 


\section{Introduction}

Gas/ice interactions are of fundamental importance to better understand the chemistry at the surface of ice-coated interstellar grain particles. ${ }^{1}$ In particular, astrochemists focus their interest on the origin and formation of amino acids in the interstellar medium. Indeed, their presence may provide information on the delivery of prebiotic molecules to the early Earth, the origin of life on Earth, and the possibility of Earth-like life elsewhere in the universe. ${ }^{2,3}$ In this respect, the methylamine $\left(\mathrm{CH}_{3} \mathrm{NH}_{2}\right)$ molecule has an important role in the chemical evolution of the simplest amino acid, glycine. The bimolecular complex of methylamine with $\mathrm{CO}_{2}$ is found to be the global minimum on the Gibbs free energy surface of the glycine isomers $\left(\mathrm{C}_{2} \mathrm{H}_{5} \mathrm{NO}_{2}\right)$, both in vacuum and in aqueous environment. ${ }^{4}$ Due to the weak intermolecular dipole - induced dipole interaction between $\mathrm{CH}_{3} \mathrm{NH}_{2}$ and $\mathrm{CO}_{2}$ this complex has a short lifetime under ambient conditions, although it can be stabilized at lower temperatures at ice surfaces. Since $\mathrm{CH}_{3} \mathrm{NH}_{2}$ is a good nucleophile and a stronger base $(\mathrm{pK}=3.36)$ than ammonia $\left(\mathrm{pK}_{\mathrm{b}}=4.75\right)$, it can react with $\mathrm{HNCO}, \mathrm{HCN}, \mathrm{HCOOH}$ and $\mathrm{CO}_{2}$ with reasonable rates, even at low temperatures in interstellar ice analogues. ${ }^{5}$ Indeed, the formation of glycine has been reported recently on interstellar ice-analog films composed of water, methylamine, and carbon dioxide under irradiation of ultraviolet (UV) photons $^{6}$ and high energy electron impacts. ${ }^{7}$ A kinetic measurement showed ${ }^{8}$ that the photochemical process resulting in glycine can be efficient at the ice surfaces since it can maintain a substantial amount of glycine, suggesting that interstellar amino acids can be formed on ice grains. ${ }^{6}$ Methylamine is detected in dense clouds ${ }^{9}$ and understood to be present on icy grains at concentrations of less than $1 \%$ relative to water. However, this is too low for its detections by astronomical infrared observations ${ }^{8}$ and no structural information is available about ice-adsorbed methylamine molecules.

As in the interstellar medium, ice surfaces are also thought to play a key role in the atmosphere of the Earth by catalyzing ozone destruction in the polar stratosphere through halogen activation ${ }^{10}$ and by partitioning organic compounds from gas to ice phases in the troposphere. ${ }^{11}$ Gas/ice interactions also possibly participate in scavenging of atmospheric pollutant molecules by falling snow. ${ }^{12}$ Amine molecules are common atmospheric species, emitted as gases from a variety of sources. ${ }^{13}$ They are also found in atmospheric condensed phases including aerosols, rain-water, and fog-water. ${ }^{14}$ More generally, amines have attracted increasing attention due to their potential role in enhancing aerosol nucleation rates in the 
lower troposphere. ${ }^{15}$ Because low-molecular weight amines are highly water-soluble, a few studies have been devoted to their interaction with liquid water in order to better characterize their likely dissolution in aqueous aerosols at high temperature, and thus their gas/particle partitionning. ${ }^{14-16}$ By contrast, very little is known about their interaction with ice surfaces as encountered in high-altitude cirrus clouds or snow flakes. As a consequence, the thermodynamic and kinetic properties of amines in the atmosphere are not well quantified and only few atmospheric models incorporate them although they are ubiquitous. ${ }^{13,14}$

In summary, in astrophysics as well as in atmospheric studies, no structural information is available about ice-adsorbed amine molecules, neither in the interstellar medium, nor in the Earth's atmosphere. This missing energetic and structural characterization can, however, be obtained by molecular scale computer simulations, ${ }^{17}$ since in this kind of calculations a detailed, atomistic level insight is obtained, within the limits of an appropriately chosen model, into the system of interest. However, in spite of the fact that methylamine has been the subject of several computer simulation investigations in the liquid phase as a neat liquid, ${ }^{18-20}$ in aqueous mixtures ${ }^{18,19,21}$ as well as the solvent of various ionic systems, ${ }^{22}$ we are not aware of such studies concerning its adsorption at the ice surface.

In the past two decades, the method of molecular dynamics (MD) simulation has been widely used to characterize the structure and energetics of the adsorption layer of various atmospheric species on ice. ${ }^{23-32}$ In addition, Grand Canonical Monte Carlo (GCMC) simulations $^{17,33}$ have also been performed to simulate the adsorption isotherms of various classes of volatile organic compounds and atmospheric pollutants on ice at low temperatures. ${ }^{34-44}$ Indeed, the GCMC method is particularly suitable for studying adsorption, because in this method the chemical potential rather than the number of the adsorbate molecules is fixed in the simulation. As a consequence, by systematically varying the value of this chemical potential in a series of GCMC simulations, and determining the number of the adsorbed molecules per surface unit as a function of this, the adsorption isotherm can be calculated directly, from extremely low pressures up to the point of condensation. A deeper analyses can always be performed for physically relevant surface coverages. In a set of systematic investigations, the adsorption isotherms of methanol, ${ }^{34}$ aldehydes, ${ }^{35,38,39}$ acetone, ${ }^{36}$ formic acid, ${ }^{37}$ aromatic hydrocarbon molecules, ${ }^{40} \mathrm{H}_{2} \mathrm{O}_{2},{ }^{41} \mathrm{HCN},{ }^{42}$ and chlorofluorocarbons ${ }^{43,44}$ on ice have been simulated so far. When possible, the comparison between simulated and 
measured isotherms showed a good agreement, giving thus confidence in the theoretical approach.

We use here the GCMC method to characterize the adsorption of methylamine at the surface of $\mathrm{I}_{\mathrm{h}}$ ice at low temperature. Indeed, besides the atmospheric and astrophysical interests alluded to above, this study aims at a more fundamental and systematic characterization of organic molecules adsorbed on ice surfaces, with a special focus on the influence of the amino group on the interaction with ice. The adsorption isotherms, the layering of the adsorbed molecules as well as the orientation and binding energy of the adsorbed molecules that are in direct contact with the ice phase are analyzed in detail.

\section{Computational Details}

In order to simulate the adsorption behavior of $\mathrm{CH}_{3} \mathrm{NH}_{2}$ at the (0001) surface of $\mathrm{I}_{\mathrm{h}}$ ice, grand canonical $(\mu, V, T)$ Monte Carlo calculations were performed at the temperature of $200 \mathrm{~K}$, characteristic of the upper troposphere, at 41 different chemical potentials of methylamine, $\mu$. The chemical potentials were chosen in such a way that the lowest $\mu$ value $(-60.47 \mathrm{~kJ} / \mathrm{mol})$ corresponds to practically no methylamine molecule in the simulation box, while the simulation at the highest value $(-27.21 \mathrm{~kJ} / \mathrm{mol})$ clearly belongs to the system having stable condensed methylamine above the ice surface. These $\mu$ values and the corresponding mean number of methylamine molecules in the basic box, $\langle N\rangle$, are tabulated in Table 1 . In all simulations, the $\mathrm{I}_{\mathrm{h}}$ crystal structure of the ice was represented by 2880 water molecules arranged in 18 layers placed in the middle of a $100 \AA \times 35.926 \AA \times 38.891 \AA$ rectangular simulation box. The largest edge of the simulation box was set as the ice surface normal, while the two smaller axes ( $Y$ and $Z$ ) were chosen in accordance with the periodicity of the $\mathrm{I}_{\mathrm{h}}$ ice crystal.

Potential of the methylamine was specified by the model proposed by Impey et al., ${ }^{22}$ while the TIP5P model ${ }^{45}$ was chosen for water due to the fact that it is able to describe the melting point of $I_{h}$ ice rather accurately. ${ }^{46,47}$ Both of these models are rigid and pairwise additive, describing the interaction of a molecule pair as the sum of Lennard-Jones and Coulomb contributions between all pairs of their interaction sites. The methylamine model treats the $\mathrm{CH}_{3}$ group as a united atom, while the TIP5P water model employs also two non- 
atomic interaction sites besides the atomic ones. The force field parameters used are collected in Tables 2 and 3. The summation of the $u_{i j}$ pair interaction energies over all molecule pairs within a center-center cut-off distance of $12.5 \AA$ provides the potential energy of the simulated system $(U)$. All interactions were neglected beyond this cut-off, which is in line with the parametrization of the TIP5P water model ${ }^{45}$.

The simulations, performed with the code $\mathrm{MMC}^{48}{ }^{48}$ were started from a configuration with a perfect $\mathrm{I}_{\mathrm{h}}, 18$ layer thin ice slab and 2 methylamine molecules having random positions in the vapor phase. A Monte Carlo simulation step consisted either in attempting a random translation (to a maximum distance of $0.25 \AA$ ) and randomly rotation (by no more than $15^{\circ}$ ) of a molecule, or attempting to change the number of methylamine molecules by one by either inserting or deleting a molecule. Molecule displacement and insertion/deletion trials were performed in alternating order. Insertions and deletions of methylamine were attempted with $50 \%$ probabilities according to the cavity biased strategy suggested by Mezei, ${ }^{49,50}$ i.e., only centers of empty cavities with a radius of at least $2.6 \AA$ were considered as possible centers for insertions. The attempted insertions and deletions were accepted or rejected according to the corresponding acceptance criterion. ${ }^{49,50}$ Searching for cavities was done along a $100 \times 100 \times 100$ grid, and repeated in every $10^{6}$ Monte Carlo steps of the simulations. The probability of finding an empty cavity for insertion, $P_{\text {cav }}$, a value needed for applying the acceptance criterion of the cavity biased scheme, ${ }^{49,50}$ was determined as the ratio of the number of suitable cavities found and total number of grid points tested. The standard Metropolis criterion was used for the acceptance or rejection of the molecular displacement steps. ${ }^{17,51}$ In this way at least $0.1 \%$ of the insertion/deletion attempts turned out to be successful, while that of the molecule displacement attempts is above $20 \%$.

After $4 \times 10^{8}-10^{9}$ Monte Carlo steps, all systems were considered as equilibrated since even the slowest systematic changes of mean number of adsorbed molecules, $\langle N\rangle$, had been eliminated. The $\langle N\rangle$ values were then determined in consecutive $10^{8}$ steps long equilibrium simulations. Furthermore, at five selected $\mu$ values (see Table 1), 2500 sample configurations per system, separated by $2 \times 10^{5}$ Monte Carlo steps, were saved for detailed structural, orientational and energetic analyses in an additional $5 \times 10^{8}$ Monte Carlo steps long run. Finally, all properties calculated were averaged over the sample configurations collected as well as over the both sides of simulated ice slab. 


\section{Results and Discussion}

3.1. Adsorption Isotherm. The mean number of methylamine molecules in the system, $\langle N\rangle$, is shown in Figure 1 as a function of its chemical potential, $\mu$; the corresponding data are also collected in Table 1. Since virtually all methylamine molecules turned out to be next to the ice surface, while the bulk vapor phase contained a negligible number of molecules in every case up to the point of condensation, the resulting $\langle N\rangle$ vs. $\mu$ data practically coincide with the adsorption isotherm. As it is seen, after a sharp exponential rise the isotherm reaches a rather long plateau, covering a roughly $5 \mathrm{~kJ} / \mathrm{mol}$ wide range of chemical potentials around the $\mu$ value of about $-40 \mathrm{~kJ} / \mathrm{mol}$. Such a plateau, observed previously, e.g., for the adsorption of methanol ${ }^{34}$ and formic acid ${ }^{37}$ on ice, typically corresponds to monolayer coverage of the solid surface and indicates that this monomolecular adsorption layer is particularly stable, presumably due to the strong lateral interaction acting between the adsorbed molecules. This point is further investigated in detail in the following sub-sections. At the end of this plateau, above the $\mu$ value of $-35 \mathrm{~kJ} / \mathrm{mol}$, the isotherm exhibits a rather sharp increase, indicating the starting of multilayer adsorption. However, this sloped part of the isotherm is very short, and it is followed by a sudden jump around $-33.5 \mathrm{~kJ} / \mathrm{mol}$, corresponding to the condensation of methylamine. Thus, although the isotherm shows traces of multilayer adsorption, the presence of at least parts of an outer molecular layer is stable only in a very narrow range of chemical potentials (and, hence, of pressures), as further building up of these outer molecular layers is prevented by the condensation of methylamine.

To further analyze the obtained adsorption isotherm we have transformed it to the more conventional $\Gamma$ vs. $p_{\text {rel }}$ form, where $\Gamma$ is the surface density of the adsorbed molecules, calculated as

$$
\Gamma=\frac{<N>}{2 Y Z},
$$

(where the factor 2 in the denominator stands for the two ice surfaces present in the basic box), and $p_{\text {rel }}=p / p_{0}$ is the relative pressure, i.e., the pressure of the system, $p$, normalized by that of the saturated vapor, $p_{0}$. Since the bulk vapor phase of the system is practically empty in every 
case, the value of the absolute pressure, $p$, can hardly be evaluated in the simulations. However, the relative pressure can simply be calculated as ${ }^{52}$

$$
p_{\text {rel }}=\frac{p}{p_{0}}=\frac{\exp \left(\mu / k_{\mathrm{B}} T\right)}{\exp \left(\mu_{0} / k_{\mathrm{B}} T\right)}
$$

where $k_{\mathrm{B}}$ stands for the Boltzmann constant, and $\mu_{\mathrm{o}}$ is the chemical potential value corresponding to the point of condensation. From the $\langle N\rangle$ vs. $\mu$ data we can estimate the value of $\mu_{\mathrm{o}}$ as $-33.41 \mathrm{~kJ} / \mathrm{mol}$. It should be noted that since $p_{\text {rel }}$ stands for the pressure of the vapor phase relative to that of the saturated vapor, the $\langle N\rangle(\mu)$ isotherm can be converted meaningfully to the $\Gamma\left(p_{\text {rel }}\right)$ form only up to the point of condensation.

The $\Gamma$ vs. $p_{\text {rel }}$ isotherm is shown in the inset of Fig. 1 and the corresponding data are included in Table 1. As it is seen, the exponential rise of the $\langle N\rangle(\mu)$ isotherm at low chemical potential values is converted to a steep linear increase of $\Gamma\left(p_{\text {rel }}\right)$. This rise turns rather quickly into an almost constant plateau in the $p_{\text {rel }}$ range of about $0.05-0.2$. This plateau covers the relative pressure range from about 0.2 to 0.9 , emphasizing the remarkably broad pressure range in which the saturated adsorption monolayer of methylamine is stable at the surface of ice. At relative pressures above 0.9 the isotherm turns again upward, indicating again that multilayer adsorption starts right below the pressure at which the condensation of methylamine occurs.

To gain further insight into the nature of this particularly stable adsorption monolayer, we have fitted a Langmuir isotherm, ${ }^{53,54}$

$$
\Gamma=\Gamma_{\max } \frac{p_{\mathrm{rel}} K}{1+p_{\mathrm{rel}} K}
$$

to the $\Gamma$ vs. $p_{\text {rel }}$ data up to the relative pressure of 0.75 (i.e., leaving out the last two data points, corresponding to multilayer adsorption). In this equation the parameter $\Gamma_{\max }$ is the surface density of the saturated monolayer and $K$ is the Langmuir partition coefficient. The best fitting curve, shown by red dashed line in Fig. 1, corresponds to the $\Gamma_{\max }$ and $K$ values of $10.35 \mu \mathrm{mol} / \mathrm{m}^{2}$ and 104.1, respectively. The fitted curve follows the data points rather accurately both in the linearly rising part and in the plateau; however, it deviates considerably from them in the turning region. The insufficient description of the data in this pressure range by a Langmuir isotherm is demonstrated in the innermost inset of Fig. 1, showing this part of 
the isotherm on a magnified scale. Since the Langmuir isotherm assumes no interaction between the adsorbed molecules, the failure of the Langmuir fit, in accordance with the observed stability of the saturated monolayer in a broad range of pressures, suggests the presence of rather strong attractive lateral interactions between the adsorbed molecules.

Based on the behavior of the adsorption isotherm, we have chosen five $\mu$ values for a thorough analysis of the structure and energetics of the adsorption layer (or the first molecular layer of methylamine in the case of a condensed phase). Thus, in system 1, corresponding to the $\mu$ value of $-47.17 \mathrm{~kJ} / \mathrm{mol}$, the ice surface is almost empty; both surfaces in the basic box host only 2-3 methylamine molecules (see Table 1). In system 2 , at $\mu=-42.18 \mathrm{~kJ} / \mathrm{mol}$ the ice surface is only partly saturated, it hosts about one third of its full capacity. System 3 at $\mu=-35.53 \mathrm{~kJ} / \mathrm{mol}$ is located at the broad plateau of the isotherm corresponding to the presence of the saturated monolayer. System 4 , at $\mu=-33.45 \mathrm{~kJ} / \mathrm{mol}$, is located right below the point of condensation, when traces of outer molecular layers of methylamine are also present. Finally, system 5 , at $\mu=-32.20 \mathrm{~kJ} / \mathrm{mol}$, is above the point of condensation, and thus it corresponds to the condensed phase of methylamine. An equilibrium snapshot of the interfacial region in systems 1-5 is shown for illustration in Figure 2, both in top and side views.

3.2. Density Profiles. The molecular number density profile of methylamine along the interface normal axis, $X$, is shown in Figure 3 for systems 1-5. For reference, the density profile of the outmost layer of the ice phase is also shown in the main figure, and the water number density profile across the entire ice phase is shown in the inset of the figure (as obtained in system 1). The positions of the methylamine and water molecules have been represented by that of their $\mathrm{N}$ and $\mathrm{O}$ atoms, respectively.

The resulting density profiles confirm the conclusions drawn from the shape of the adsorption isotherm. Thus, systems 1 and 2 are characterized by unsaturated monolayers, the corresponding density profiles show a single peak around $33.5 \AA$, i.e., in contact with the ice phase. The profile of system 3 still consists of this single peak, which now reaches its full height. System 3, located in the plateau region of the isotherm (Fig. 1), is characterized by a saturated adsorption monolayer, which turns out to be stable in a broad pressure range. The building up of outer molecular layers starts in system 4, just below the point of condensation, as seen from the second peak of the profile, around $37 \AA$, and an additional shoulder around 
$39 \AA$ A. Finally, in system 5 the profile does not drop to zero, indicating the presence of a condensed phase of methylamine.

It is interesting to note that the density profile obtained in system 5 does not resemble that of a liquid phase. It shows a number of maxima of various heights, separated always by 2.5-3 $\AA$ from each other rather than a damped oscillation rapidly converging to the bulk phase density value. This density profile suggests that the condensed methylamine phase is in a glassy state rather than a liquid. To understand this behavior one should consider the fact that the experimental triple point temperature of methylamine is $180 \mathrm{~K},{ }^{55}$ which is rather close to the temperature of our simulations of $200 \mathrm{~K}$. Considering also that the formation of a crystalline phase in a computer simulation typically requires a run several orders of magnitude longer than we performed here, we believe that what we see is a precursor of crystal formation, and that the system simulated is thus likely below its triple point temperature.

In the following we analyze the orientations and energetics of the adsorbed methylamine molecules that are in a direct contact with the ice phase. The outer boundary of this first molecular layer can be conveniently defined through the density profile obtained in system 5 as the position of its first minimum. This minimum, located at the $X$ value of $35.4 \AA$, is shown by the dotted vertical line in Fig. 3 .

3.3. Orientational Preferences of the Adsorbed Methylamine Molecules. We have already shown that the angular polar coordinates of the external vector, $\vartheta$ and $\phi$, in a local Cartesian frame $(x, y, z)$ fixed to the individual rigid bodies is an eligible choice for describing the relative orientation of rigid body, given that $\cos \vartheta$ and $\phi$ are regarded as the independent orientational variables. ${ }^{56,57}$ In order to get information about the preferred orientations of the adsorbed methylamines in this work, the external vector is defined by the surface normal vector, pointing away from the ice phase $(\underline{X})$, while methylamine molecules are considered as the rigid bodies, and their local Cartesian frame is defined in the following way: the $\mathrm{N}$ atom is its origin, the $\mathrm{N}-\mathrm{C}$ bond corresponds to axis $z$ (where the unit vector $z$ points to the $\mathrm{CH}_{3}$ group), axis $y$ is parallel with the line connecting the two $\mathrm{H}$ atoms of the $\mathrm{NH}_{2}$ group, and axis $x$ is perpendicular to the above two, directed in such a way that the $x$ coordinates of the two $\mathrm{NH}_{2}$ hydrogen atoms are positive. Thus, $\vartheta$ is the angle between axis $z$ of this frame and the surface normal vector, $\underline{X}$, while $\phi$ is the angle of axis $x$ and the projection of $\underline{X}$ to the $x y$ plane of this local frame, as shown in Figure 4. Due to the fact that the methylamine model used is 
symmetric, this local frame can always be defined in such a way that the polar angle $\phi$ does not exceed $180^{\circ}$.

The $P(\cos \vartheta, \phi)$ orientational maps obtained in the first methylamine adlayer in systems 1, 2, 3, and 5 are shown in Figure 5. The map obtained in system 4 shows very similar features to that in system 3, hence it is omitted from this figure. As it is seen, in systems 1-3 orientations corresponding to $\cos \vartheta$ values of nearly 0 occur with high probabilities. In these orientations the $\mathrm{N}-\mathrm{CH}_{3}$ bond is parallel with the ice surface. In system 1 the most probable orientation corresponds to a $\phi$ value of $90^{\circ}$, i.e., one of the two $\mathrm{NH}_{2}$ hydrogen atoms pointing towards and the other one away from the ice surface. The peak of this preferred orientation, marked here as orientation A, broadens considerably toward lower $\cos \vartheta$ values, corresponding to a small tilt of the molecule away from the above orientation in such a way that its $\mathrm{CH}_{3}$ group as well as the lone pair of the $\mathrm{N}$ atom point flatly towards the surface. In this orientation, illustrated also in Fig. 5, the methylamine molecule can form up to two H-bonds with water molecules at the ice surface that are aligned in one of their four preferred orientations: ${ }^{34}$ one by donating the downward oriented $\mathrm{H}$ atom of the $\mathrm{NH}_{2}$ group, and another one by accepting a water $\mathrm{H}$ atom in the direction of the slightly downward oriented lone pair.

As the ice surface comes closer to saturation, the preference for alignment A becomes progressively weaker. In the case of the saturated monolayer (system 3 ) it splits and moves to $\phi$ values of $120^{\circ}$ and $60^{\circ}$. These orientations are marked as A1 and A2. The orientations corresponding to $\phi$ values of $180^{\circ}$ and $0^{\circ}$, marked here as B (traces of which are already seen in system 1) and C, respectively, become progressively more populated. It is also seen that the preferred orientations move to slightly negative $\cos \vartheta$ values as the adsorption layer gets closer to saturation, indicating a slight tilt of the $\mathrm{N}-\mathrm{CH}_{3}$ bond towards the ice surface. In orientation A1 one of the two $\mathrm{NH}_{2}$ hydrogen atoms points as straight towards the ice surface, while in A2 as straight away from the ice surface as possible within the constraint imposed by the preferred (parallel with the surface) orientation of the $\mathrm{N}_{-} \mathrm{CH}_{3}$ bond. In orientations $\mathrm{B}$ and $\mathrm{C}$ the two $\mathrm{N}-\mathrm{H}$ bonds of the $\mathrm{NH}_{2}$ group tilt equally from the surface, the two $\mathrm{H}$ atoms pointing towards the ice phase in orientation B and away from it in orientation C. All preferred orientations are also illustrated in Fig. 5. It should be noted that a methylamine molecule that is in orientation A2 or B can form, in principle, up to two hydrogen bonds with surface water molecules aligned in one of their preferred interfacial orientations: ${ }^{34}$ in the first case through the downward oriented 
$\mathrm{H}$ atom and via the lone pair while in the second case by both $\mathrm{NH}_{2}$ hydrogen atoms. By contrast, only one such hydrogen bond can be formed in orientations A1 and C: in the first case by the downward oriented $\mathrm{H}$ atom while in the second case by the lone pair. Typical hydrogen bonding arrangements between adsorbed methylamine and surface water molecules, in which all molecules are aligned in one of their preferred orientations are shown in Figure 6.a.

In system 5 the peaks $\mathrm{A} 1$ and $\mathrm{A} 2$ shift to the $\cos \vartheta$ value of -1 . This value corresponds to the orientation in which the $\mathrm{CH}_{3}$ group of the methylamine molecule points straight towards the ice phase. In this case, the polar angle $\phi$ loses its meaning (as the projection of vector $\underline{X}$ to the $x y$ plane of the local Cartesian frame becomes a point). In this orientation, marked as D and also illustrated in Fig. 5, no hydrogen bond can be formed between the methylamine molecule and the surface waters, since the polar group of methylamine points straight away from the ice phase. This orientation is made possible by the presence of highly populated outer methylamine layers in system 5, containing condensed methylamine.

Finally it should be noted that the preferred methylamine orientations in the first layer are such that they do not correspond to the alignments required for a hydrogen bonded methylamine pair. On the other hand, considering the fact that the dipole vector of the methylamine molecule coincides more or less with the bisector of the $\mathrm{H}-\mathrm{N}-\mathrm{H}$ angle, neighboring adsorbed methylamine molecules can easily form strongly interacting dipoledipole pairs in these orientations, either in a head-to-tail or in an antiparallel relative arrangement. Strong dipolar interactions between neighboring methylamine molecules can further rationalize the observed orientational preferences. Thus, the dipole vectors of two neighboring molecules, both aligned in orientation A, can easily be arranged in a head-to-tail fashion. Similar but weaker, zig-zagged head-to-tail dipolar alignments can also be formed by such pairs in any other preferred surface orientations. Furthermore, orientations corresponding to symmetric positions along the $\phi$ axis of the orientational map (i.e., A1 and A2, B and C) correspond to opposite dipolar alignments. Therefore, neighboring methylamine molecules aligned in such pairs of orientations can easily form antiparallel dipolar pairs. Finally, the preference for alignment $\mathrm{D}$ at high surface coverages can also be rationalized considering dipolar attraction within the first molecular layer. At low surface coverages orientation D is evidently not populated because of the lack of possible hydrogen bonds with the surface waters. However, at high surface coverages methylamine molecules that fit to the first 
molecular layer in this alignment can form head-to-tail dipolar pairs with neighbors of orientations A2 or $\mathrm{C}$, and antiparallel dipolar pairs with neighbors of orientations A1 or B. Typical relative arrangements of two neighboring adsorbed methylamine molecules, corresponding to a strong dipolar attraction between them, in which both molecules are aligned in one of their preferred orientations, are illustrated in Figure 6.b.

3.4. Adsorption Energy. The distribution of the binding energy, $U_{\mathrm{b}}$, is calculated for the first methylamine adlayer in systems 1-5 in order to understand the energetics of the adsorption process. $U_{\mathrm{b}}$ is the total interaction energy of an adsorbed methylamine molecule with the rest of the system. Furthermore, interaction energy distribution of a molecule in the first methylamine adlayer with the ice phase, $U_{\mathrm{b}}^{\text {ice }}$, and with the other methylamine molecules, $U_{\mathrm{b}}^{\text {lat }}$, are calculated.

The above mentioned energy distributions obtained in systems 1, 2, 3 and 5 are shown in Figure 7. The distributions obtained in system 4 turned out to be very similar to those of system 3 , therefore the corresponding curves are not shown here. In system $1 P\left(U_{\mathrm{b}}^{\text {ice }}\right)$ is a unimodal distribution, having its highest probabilities around $-55 \mathrm{~kJ} / \mathrm{mol}$. Since the binding energy of a hydrogen bond is about $20-25 \mathrm{~kJ} / \mathrm{mol}$, it can be interpreted as the majority of the adsorbed methylamines indeed form two H-bonds with the surface waters in system 1. It is also worthy to mention that the heat of adsorption at infinitely low surface coverage can be estimated as $-51.3 \mathrm{~kJ} / \mathrm{mol}$ from the mean value of this distribution. Bimodal $P\left(U_{\mathrm{b}}^{\text {ice }}\right)$ distribution is observed in the case of system 2; its main peak occurs around $-35 \mathrm{~kJ} / \mathrm{mol}$ and the another peak can be found around $-50 \mathrm{~kJ} / \mathrm{mol}$. As the first molecular layer saturates, the peak at $-35 \mathrm{~kJ} / \mathrm{mol}$ becomes the only feature of the distribution, and it shifts to slightly higher energies around $-30 \mathrm{~kJ} / \mathrm{mol}$ in systems $3-5$. This can be explained that, as the first molecular layer gets progressively closer to saturation, an increasing fraction of the adsorbed methylamine molecules form only one $\mathrm{H}$-bond with the surface waters. In the case of a fully saturated adsorption layer the fraction of the double hydrogen bonded methylamine molecules vanishes. Furthermore, the $P\left(U_{\mathrm{b}}^{\text {ice }}\right)$ distributions shift slightly to higher energies with increasing surface coverage, indicating that, presumably due to the increasing competition between the adsorbed 
molecules, these methylamine-water hydrogen bonds become progressively weaker. Finally, in the case of system 5, a minor mode occurs at the high energy side of the major mode of $P\left(U_{\mathrm{b}}^{\text {ice }}\right)$, around $-8 \mathrm{~kJ} / \mathrm{mol}$. This shoulder corresponds to methylamine molecules that form no hydrogen bonds with the surface waters, and give rise to peak D of the orientational map.

The $P\left(U_{\mathrm{b}}^{\text {lat }}\right)$ distribution obtained in system 1 is dominated by a very high and narrow peak at zero energy. This peak reflects the fact that at such a low surface coverage the majority of the adsorbed molecules are separated from each other and their lateral interaction is thus negligible. However, besides this trivial peak another small one is apparent slightly above $-20 \mathrm{~kJ} / \mathrm{mol}$. This peak can be attributed to neighboring pairs of adsorbed methylamine molecules that strongly interact with each other. The fact that the position of this peak is located above $-20 \mathrm{~kJ} / \mathrm{mol}$ excludes the possibility of hydrogen bond formation, in accordance with the conclusion drawn from the orientational analysis. However, this interaction energy is certainly low enough for a molecule pair with strong dipolar attraction. As the surface gets progressively closer to saturation the trivial peak of the isolated molecules at zero energy decreases and finally vanishes in system 3. Simultaneously, the other peak increases, broadens and shifts to lower energies: in systems 2,3 and 5 it appears at about $-20,-30$, and $-40 \mathrm{~kJ} / \mathrm{mol}$, respectively. This shift reflects the increasing number of neighbors within the adsorption monolayer and, reaching the condensed system 5, also beneath this layer.

The total binding energy distribution, $P\left(U_{\mathrm{b}}\right)$, is unimodal in every case; the position of its peak shifts to lower energies with increasing surface coverage, being around -50, -55, -60, and $-72 \mathrm{~kJ} / \mathrm{mol}$ in systems $1,2,3$, and 5, respectively. This progressive shift reflects the fact that the increasing lateral interaction, resulting from the increasing number of adsorbed molecules, clearly overcompensates the weakening of the adsorbate-ice interaction caused by the increasing competition of the methylamine molecules.

\section{Conclusions}

In this paper we have presented a detailed analysis of the adsorption of methylamine at the (0001) surface of $I_{h}$ ice under tropospheric conditions on the basis of a series of grand canonical ensemble Monte Carlo computer simulations. The adsorption isotherm exhibits a plateau corresponding to a saturated monomolecular adsorption layer, indicating that such a 
monolayer is stable in a remarkably broad range of chemical potentials, and hence of pressures. At pressures close to that of the saturated vapor, traces of multilayer adsorption have been detected; however, the building up of outer molecular layers is prevented by the condensation of methylamine.

The remarkable stability of the saturated adsorption monolayer results from a delicate interplay between hydrogen bonding (between surface water and adsorbed methylamine molecules), and strong dipolar attraction (between the neighboring adsorbed molecules). As a consequence, the adsorption isotherm deviates noticeably from the Langmuir shape and the adsorption process is accompanied by rather large energy changes. Thus, the heat of adsorption at infinitely low surface coverages is estimated to be $-51.3 \mathrm{~kJ} / \mathrm{mol}$, the interaction energy of an isolated neighboring adsorbate pair is found to be about $-20 \mathrm{~kJ} / \mathrm{mol}$, and the average total binding energy of an adsorbed molecule turns out to be in the range of $-50--60 \mathrm{~kJ} / \mathrm{mol}$, even in the lack of outer adsorption layers. Correspondingly, the adsorbed methylamine molecules exhibit rather rich orientational preferences. At low surface coverages these preferences are mainly governed by the requirement of maximizing the number of hydrogen bonds formed with the surface water molecules, while with increasing surface coverages the formation of favorable (i.e., antiparallel or head-to-tail type) dipolar relative near-neighbor alignments becomes also an increasingly important factor in this respect.

From a more general, atmospheric, point of view, amines are among the few atmospheric bases which, in competition with ammonia, have a unique acid-neutralizing capacity. ${ }^{13}$ Regarding the importance of a better quantification of their gas/particle partitioning, the present calculations show that methylamine strongly interacts with the surface of ice at the low temperatures typical of the upper troposphere. Thus, ice particles in cirrus clouds may trap significant fractions of such molecules, participating in their scavenging from the gas phase, and, as a consequence, modifying their potential role in particle nucleation and secondary organic aerosol formation.

In the same way, the large interaction energies with ice calculated here are consistent with the trapping of methylamine by solid water at the surface of interstellar grains or in comets, ${ }^{58}$ where subsequent chemical/photochemical reactions may well participate in the formation of glycine, i.e., the simplest amino acid molecule involved in prebiotic chemistry. Indeed, very recent mass spectrometer measurements have evidenced the presence of glycine accompanied by methylamine and ethylamine in the coma of comet 67P/Churyumov- 
Gerasimenko, indicating that these three molecules are closely related to each other in the ice mantle of the comet. ${ }^{59}$ This discovery stresses the urgent need for a better understanding of the chemistry at the ice surface in the interstellar medium, to which the present study can participate by giving, for instance, a detailed picture of the adsorbed geometries on ice. Indeed, one important finding of our calculations is that, at low coverage, the methylamine molecule is adsorbed with its $\mathrm{N}-\mathrm{CH}_{3}$ axis parallel to the ice surface, with at least one hydrogen atom of the amine group involved in hydrogen bonding with one surrounding water molecule. Such $\mathrm{H}-$ bonding has been shown to be required for the barrier-free formation of a glycine precursor, namely the methylcarbamic acid zwitterion $\left(\mathrm{CH}_{3} \mathrm{NH}_{2}{ }^{+} \mathrm{COO}^{-}\right)$, as resulting from the reaction between methylamine and carbon dioxide in interstellar ices. ${ }^{3}$

Finally, from a more fundamental point of view, the present study adds a new class of organic molecules in our systematic characterization of the adsorption isotherms of atmospheric species on ice. The evidence of a very stable monolayer of methylamine molecules on ice could motivate new experiments for supporting our conclusions.

\section{Acknowledgements}

The authors acknowledge financial support from the NKFIH Foundation, Hungary (project number 119732) from the Hungarian-French TéT (BALATON) program (project number TéT_15_FR-1-2016-0056), and from CNRS in the frame of the PICS program. Milán Szőri was supported by a Magyary Zoltán fellowship within the framework of TÁMOP 4.2.4.A/2-11-1-2012-0001 (A2-MZPD-12-0139) and he is currently János Bolyai Research Scholar of the Hungarian Academy of Sciences (BO/00113/15/7). 


\section{References}

(1) Ehrenfreund, P.; Fraser, H. J.; Blum, J.; Cartwright, J. H. E.; García-Ruiz, J. M.; Hadamcik, E.; Levasseur-Regourd, A.C.; Price, S.; Prodi, F.; Sarkissian, A. Physics and Chemistry of Icy Particles in the Universe: Answers from Microgravity. Planet. Space Sci. 2003, 51, 473-494.

(2) Newton, D.E. Chemistry of Space; Facts on File Inc.: New York, 2007.

(3) Kayi, H.; Kaiser, R. I.; Head, J. D. A Computational Study on the Structures of Methylamine-Carbon Dioxide-Water Clusters: Evidence for the Barrier Free Formation of the Methylcarbamic Acid Zwitterion $\left(\mathrm{CH}_{3} \mathrm{NH}_{2}{ }^{+} \mathrm{COO}^{-}\right)$in Interstellar Water Ices. Phys. Chem. Chem. Phys. 2011, 13, 11083-11098.

(4) Szőri, M.; Jójárt, B.; Izsák, R.; Szőri, K.; Csizmadia, I. G.; Viskolcz, B. Chemical Evolution of Biomolecule Building Blocks. Can Thermodynamics Explain the Accumulation of Glycine in the Prebiotic Ocean? Phys. Chem. Chem. Phys., 2011, 13, 7449-7458.

(5) Vinogradoff, V.; Duvernay, F.; Danger, G.; Theulé, P.; Borget, F.; Chiavassa, T. Formaldehyde and Methylamine Reactivity in Interstellar Ice Analogues As a Source of Molecular Complexity at Low Temperature. Astron. Astrophys., 2013, 549, A40.

(6) Lee, C. W.; Kim, J. K.; Moon, E. S.; Minh, Y. C.; Kang, H. Formation of Glycine on Ultraviolet-Irradiated Interstellar Ice-Analog Films and Implications for Interstellar Amino Acids. Astrophys. J., 2009, 697, 428-435.

(7) Holtom, P. D.; Bennett, C. J.; Osamura, Y.; Mason, N. J.; Kaiser, R. I. A Combined Experimental and Theoretical Study on the Formation of the Amino Acid Glycine $\left(\mathrm{NH}_{2} \mathrm{CH}_{2} \mathrm{COOH}\right)$ and Its Isomer $\left(\mathrm{CH}_{3} \mathrm{NHCOOH}\right)$ in Extraterrestrial Ices. Astrophys. J., 2005, 626, 940-952.

(8) Bossa, J.-B.; Duvernay, F.; Theulé, P.; Borget, F.; d'Hendecourt, L.; Chiavassa, T. Methylammonium Methylcarbamate Thermal Formation in Interstellar Ice Analogs: A 
Glycine Salt Precursor in Protostellar Environments. Astron. Astrophys., 2009, 506, $601-608$.

(9) Ziurys, L. M.; Adande, G. R.; Edwards, J. L.; Schmidt, D. R.; Halfen, D. T.; Woolf, N. J. Prebiotic Chemical Evolution in the Astrophysical Context. Orig. Life Evol. Biosph., 2015, 45, 275-288.

(10) Solomon, S.; Garcia, R. R.; Rowland, F. S.; Wuebbles, D. J. On the Depletion of Antarctic Ozone. Nature 1986, 321, 755-758.

(11) Abbatt, J. P. D. Interactions of Atmospheric Trace Gases with Ice Surfaces: Adsorption and Reaction. Chem. Rev. 2003, 103, 4783-4800.

(12) Lei, Y. D. Wania, F. Is Rain or Snow a More Efficient Scavenger of Organic Chemicals? Atmos. Environ. 2004, 38, 3557-3571.

(13) Ge, X.; Wexler, A. S.; Clegg, S.L. Atmospheric Amines - Part I. A Review. Atmos. Environ. 2011, 45, 524-546.

(14) Ge, X.; Wexler, A. S.; Clegg, S. L. Atmospheric Amines - Part II. Thermodynamic Properties and Gas/Particle Partitioning. Atmos. Environ. 2011, 45, 561-577.

(15) Yu, F.; Luo. G. Modeling of Gaseous Methylamines in the Global Atmosphere: Impacts of Oxidation and Aerosol Uptake. Atmos. Chem. Phys. 2014, 14, 12455-12464.

(16) Mmereki, B. T.; Hicks, J. M.; Donaldson, D. J. Adsorption of Atmospheric Gases at the Air-Water Interface. 3: Methylamines. J. Phys. Chem. A 2000, 104, 10789-10793.

(17) Allen, M. P.; Tildesley, D. J. Computer Simulation of Liquids; Clarendon: Oxford, 1987.

(18) Rizzo, R. C.; Jorgensen, W. L. OPLS All-Atom Model for Amines: Resolution of the Amine Hydration Problem. J. Am. Chem. Soc. 1999, 121, 4827-4836.

(19) Kusalik, P. G.; Bergman, D.; Laaksonen, A. The Local Structure in Liquid Methylamine and Methylamine-Water Mixtures. J. Chem. Phys. 2000, 113, 8036-8046.

(20) Kosztolányi, T.; Bakó, I.; Pálinkás, G. Hydrogen Bonding in Liquid Methanol, Methylamine, and Methanethiol Studied by Molecular-Dynamics Simulations. J. Chem. Phys. 2003, 118, 4546-4555. 
(21) Hoehn, R. D.; Carignano, M.; Kais, S.; Zhu, C.; Zhong, J.; Zeng, X. C.; Francisco, J. S.; Gladich, I. Hydrogen Bonding and Orientation Effects on the Accommodation of Methylamine at the Air-Water Interface. J. Chem. Phys. 2016, 144, 214701-1-15

(22) Impey, R. W.; Sprik, M.; Klein, M. L. Ionic Solvation in Nonaqueous Solvents: The Structure of $\mathrm{Li}^{+}$and $\mathrm{Cl}^{-}$in Methanol, Ammonia, and Methylamine. J. Am. Chem. Soc. 1987, 109, 5900-5904.

(23) Picaud, S; Hoang, P. N. M. Adsorption of Acetone Molecules on Proton Ordered Ice. A Molecular Dynamics Study. J. Chem. Phys. 2000, 112, 9898-9908.

(24) Mitlin, S., Lemak, A.S.; Torrie, B.H.; Leung, K.T. Surface Adsorption and Trapping of Xe on Hexagonal Ice at $180 \mathrm{~K}$ by Molecular Dynamics Simulations. J. Phys. Chem. B 2003, 107, 9958-9963.

(25) Picaud, S.; Hoang, P. N. M. Molecular Dynamics Simulations of Chloroform on Ice. Phys. Chem. Chem. Phys. 2004, 6, 1970-1974.

(26) Picaud, S.; Hoang, P. N. M.; Peybernès, N.; Le Calvé, S.; Mirabel, Ph. Adsorption of Acetic Acid on Ice. Experiments and Molecular Dynamics Simulations. J. Chem. Phys. 2005, 122, 194707:1-10.

(27) Dominé, F.; Cincinelli, A.; Bonnaud, E.; Martellini, T.; Picaud, S. Adsorption of Phenanthrene on Natural Snow. Environ. Sci. Technol. 2007, 41, 6033-6038.

(28) Darvas, M.; Picaud, S.; Jedlovszky, P. Molecular Dynamics Simulation of the Adsorption of Oxalic Acid on Ice Surface. Chem. Phys. Chem. 2010, 11, 3971-3979.

(29) Petitjean, M.; Darvas, M.; Le Calvé, S.; Jedlovszky, P.; Picaud, S. Adsorption of Hydroxyacetone on Pure Ice Surfaces. Chem. Phys. Chem. 2010, 11, 3921-3927.

(30) Liyana-Arachchi, T. P.; Valsaraj, K. T.; Hung, F. R. Adsorption of Naphthalene and Ozone on Atmospheric Air/Ice Interfaces. J. Phys. Chem. A 2011, 115, 9226-9236.

(31) Chen, J.; Ehrenhauser, F.; Liyana-Arachchi, T. P.; Hung, F. R.; Wornat, M. J.; Valsaraj, K. T. Adsorption of Gas-Phase Phenanthrene on Atmospheric Water and Ice Films. Polycycl. Arom. Comp. 2011, 31, 201-226. 
(32) Habartová, A.; Hormain, L.; Pluhařová, E.; Briquez, S.; Monnerville, M.; Toubin, C.; Roeselová, M. Molecular Simulations of Halomethanes at the Air/Ice Interface. J. Phys. Chem. A 2015, 119, 10052-10059.

(33) Adams, D. J. Grand Canonical Ensemble Monte Carlo for a Lennard-Jones Fluid. Mol. Phys. 1975, 29, 307-311.

(34) Jedlovszky, P.; Partay, L.; Hoang, P. N. M.; Picaud, S.; von Hessberg, P.; Crowley, J. N. Determination of the Adsorption Isotherm of Methanol on the Surface of Ice. An Experimental and Grand Canonical Monte Carlo Simulation Study. J. Am. Chem. Soc. 2006, 128, 15300-15309.

(35) Hantal, G.; Jedlovszky, P.; Hoang, P.N.M.; Picaud, S. Calculation of the Adsorption Isotherms of Formaldehyde on Ice by Grand Canonical Monte Carlo simulation. J. Phys. Chem. C 2007,111, 14170-14178.

(36) Hantal, G.; Jedlovszky, P.; Hoang, P.N.M.; Picaud, S. Investigation of the Adsorption Behavior of Acetone at the Surface of Ice. A Grand Canonical Monte Carlo Simulation Study. Phys. Chem. Chem. Phys. 2008, 10, 6369-6380.

(37) Jedlovszky, P.; Hantal, G.; Neurohr, K.; Picaud, S.; Hoang, P.N.M.; von Hessberg, P.; Crowley, J. Adsorption Isotherm of Formic Acid on the Surface of Ice, as Seen From Experiment and Grand Canonical Monte Carlo Simulations. J. Phys. Chem. C 2008, $112,8976-8987$.

(38) Petitjean, M.; Hantal, Gy.; Chauvin, C.; Mirabel, P.; Le Calvé, S.; Hoang, P. N. M.; Picaud, S.; Jedlovszky, P. Adsorption of Benzaldehyde at the Surface of Ice, Studied by Experimental Method And Computer Simulation. Langmuir 2010, 26, 9596-9606.

(39) Darvas, M.; Lasne, J.; Laffon, C.; Parent, P.; Picaud, S.; Jedlovszky, P. Adsorption of Acetaldehyde on Ice as Seen From Computer Simulation and Infrared Spectroscopy Measurements. Langmuir 2012, 28, 4198-4207.

(40) Mészár, Zs. E.; Hantal, Gy.; Picaud, S.; Jedlovszky, P. Adsorption of Aromatic Hydrocarbon Molecules at the Surface of Ice, As Seen by Grand Canonical Monte Carlo Simulation. J. Phys. Chem. C 2013, 117, 6719-6729. 
(41) Picaud, S.; Jedlovszky, P. Adsorption of $\mathrm{H}_{2} \mathrm{O}_{2}$ at the Surface of $\mathrm{I}_{\mathrm{h}}$ Ice, as Seen from Grand Canonical Monte Carlo Simulations. Chem. Phys. Letters 2014, 600, 73-78.

(42) Szőri, M.; Jedlovszky, P. Adsorption of HCN at the Surface of Ice. A Grand Canonical Monte Carlo Simulation Study. J. Phys. Chem. C 2014, 118, 3599-3609.

(43) Sumi, I.; Picaud, S.; Jedlovszky, P. Adsorption of Methylene Fluoride and Methylene Chloride at the Surface of Ice under Tropospheric Conditions. A Grand Canonical Monte Carlo Simulation Study. J. Phys. Chem. C 2015, 119, 17243-17252.

(44) Sumi, I.; Picaud, S.; Jedlovszky, P. Adsorption of Fluorinated Methane Derivatives at the Surface of Ice under Tropospheric Conditions as Seen from Grand Canonical Monte Carlo Simulations. J. Phys. Chem. C 2016, 120, 17386-17399.

(45) Mahoney, M.; Jorgensen, W. L. A Five-Site Model for Liquid Water and the Reproduction of The Density Anomaly by Rigid, Nonpolarizable Potential Functions. $J$. Chem. Phys. 2000, 112, 8910-8922.

(46) Nada, H.; van der Eerden, J. P. M. J. An Intermolecular Potential Model for the Simulation of Ice and Water near the Melting Point: A Six-Site Model of $\mathrm{H}_{2} \mathrm{O} . J$. Chem. Phys. 2003, 118, 7401-7413.

(47) Vega, C.; Sanz, E.; Abascal, J. L. F. The Melting Temperature of the Most Common Models of Water. J. Chem. Phys. 2005, 122, 114507-1-9.

(48) Mezei, M. MMC: Monte Carlo Program for Simulation of Molecular Assemblies. URL: http://inka.mssm.edu/ mezei/mmc.

(49) Mezei, M. A Cavity-Biased (T, V, $\mu$ ) Monte Carlo Method for the ComputerSimulation of Fluids. Mol. Phys. 1980, 40, 901-906.

(50) Mezei, M. Mol. Phys. Grand Canonical Ensemble Monte Carlo Study of Dense Liquid Lennard-Jones, Soft Spheres and Water. 1987, 61, 565-582. Erratum: 1989, 67, $1207-$ 1208.

(51) Metropolis, N.; Rosenbluth, A. W.; Rosenbluth, M. N.; Teller, A. H.; Teller, E. Equation of State Calculations by Fast Computing Machines J. Chem. Phys. 1953, 21 1087-1093.

(52) Daub, C. D.; Patey G. N.; Jack, D. B.; Sallabi A. K. Monte Carlo Simulations of the Adsorption of $\mathrm{CO}_{2}$ on the $\mathrm{MgO}(100)$ Surface. J. Chem. Phys. 2006, 124, 114706-1-9. 
(53) Langmuir, I. The Constitution and Fundamental Properties of Solids and Liquids. Part I: Solids. J. Am. Chem. Soc. 1916, 38, 2221-2295.

(54) Shaw, D. J. Introduction to Colloid and Surface Chemistry, Butterworths: London, 1980.

(55) Aston, J. G.; Siller, C. W; Messerly, G. H. Heat Capacities and Entropies of Organic Compounds III. Methylamine from 11.5 K to the Boiling Point. Heat of Vaporization and Vapor Pressure. The Entropy from Molecular Data. J. Am. Chem. Soc., 1937, 59, 1743-1751.

(56) Jedlovszky, P.; Vincze, Á.; Horvai, G. New Insight into the Orientational Order of Water Molecules at the Water/1,2-Dichloroethane Interface: A Monte Carlo Simulation Study. J. Chem. Phys. 2002, 117, 2271-2280.

(57) Jedlovszky, P.; Vincze, Á.; Horvai, G. Full Description of the Orientational Statistics of Molecules Near to Interfaces. Water at the Interface with $\mathrm{CCl}_{4}$. Phys. Chem. Chem. Phys. 2004, 6, 1874-1879.

(58) Ehrenfreund, P.; Charnley, S. B. Organic Molecules in the Interstellar Medium, Comets, and Meteorites. Ann. Rev. Astron. Astrophys. 2000, 38, 427-483.

(59) Altwegg, K.; Balsiger, H.; Bar-Nun, A.; Berthelier, J. J.; Bieler, A.; Bochsler, P., Briois, C.; Calmonte, U.; Combi, M. R.; Cottin, H.; et al. Prebiotic Chemicals - Amino Acid and Phosphorus - in the Coma of Comet 67P/Churyumov-Gerasimenko. Sci. Adv. 2016, 2, e1600285. 


\section{Tables}

Table 1. Data Corresponding to the Simulated Adsorption Isotherm

\begin{tabular}{|c|c|c|c|}
\hline$\mu / \mathrm{kJ} \mathrm{mol}^{-1}$ & $\langle N\rangle$ & $p / p_{0}$ & 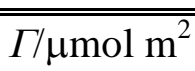 \\
\hline-60.47 & 0.0070 & $8.55 \times 10^{-8}$ & $4.16 \times 10^{-4}$ \\
\hline-59.64 & 0.0055 & $1.41 \times 10^{-7}$ & $3.27 \times 10^{-4}$ \\
\hline-58.81 & 0.0104 & $2.32 \times 10^{-7}$ & $6.18 \times 10^{-4}$ \\
\hline-57.98 & 0.0172 & $3.83 \times 10^{-7}$ & $1.02 \times 10^{-3}$ \\
\hline-57.14 & 0.0405 & $6.32 \times 10^{-7}$ & $2.41 \times 10^{-3}$ \\
\hline-56.31 & 0.0657 & $1.04 \times 10^{-6}$ & $3.90 \times 10^{-3}$ \\
\hline-55.48 & 0.0994 & $1.72 \times 10^{-6}$ & $5.91 \times 10^{-3}$ \\
\hline-54.65 & 0.0815 & $2.83 \times 10^{-6}$ & $4.84 \times 10^{-3}$ \\
\hline-53.82 & 0.2217 & $4.67 \times 10^{-6}$ & $1.32 \times 10^{-2}$ \\
\hline-52.99 & 0.3634 & $7.69 \times 10^{-6}$ & $2.16 \times 10^{-2}$ \\
\hline-52.16 & 0.6004 & $1.27 \times 10^{-5}$ & $3.57 \times 10^{-2}$ \\
\hline-51.32 & 0.7782 & $2.09 \times 10^{-5}$ & $4.62 \times 10^{-2}$ \\
\hline-50.49 & 1.206 & $3.45 \times 10^{-5}$ & $7.17 \times 10^{-2}$ \\
\hline-49.66 & 1.834 & $5.69 \times 10^{-5}$ & 0.109 \\
\hline-48.83 & 2.419 & $9.37 \times 10^{-5}$ & 0.144 \\
\hline-48.00 & 3.699 & $1.55 \times 10^{-4}$ & 0.220 \\
\hline$-47.17^{\mathrm{a}}$ & 5.089 & $2.55 \times 10^{-4}$ & 0.302 \\
\hline-46.34 & 7.273 & $4.20 \times 10^{-4}$ & 0.432 \\
\hline-45.50 & 9.919 & $6.93 \times 10^{-4}$ & 0.589 \\
\hline-44.67 & 13.89 & $1.14 \times 10^{-3}$ & 0.825 \\
\hline-43.84 & 23.34 & $1.88 \times 10^{-3}$ & 1.39 \\
\hline-43.01 & 30.72 & $3.10 \times 10^{-3}$ & 1.83 \\
\hline$-42.18^{b}$ & 54.58 & $5.12 \times 10^{-3}$ & 3.24 \\
\hline-41.35 & 80.71 & $8.44 \times 10^{-3}$ & 4.80 \\
\hline-40.52 & 112.4 & $1.39 \times 10^{-2}$ & 6.68 \\
\hline-39.69 & 133.3 & $2.29 \times 10^{-2}$ & 7.92 \\
\hline-38.85 & 145.7 & $3.78 \times 10^{-2}$ & 8.66 \\
\hline-38.02 & 152.0 & $6.24 \times 10^{-2}$ & 9.03 \\
\hline-37.19 & 155.0 & 0.103 & 9.21 \\
\hline-36.36 & 160.3 & 0.169 & 9.52 \\
\hline$-35.53^{c}$ & 163.0 & 0.279 & 9.68 \\
\hline-34.70 & 168.5 & 0.461 & 10.0 \\
\hline-33.87 & 173.5 & 0.760 & 10.3 \\
\hline-33.53 & 180.3 & 0.928 & 10.7 \\
\hline$-33.45^{\mathrm{d}}$ & 188.5 & 0.975 & 11.2 \\
\hline-33.37 & 772.3 & & \\
\hline$-33.20^{\mathrm{e}}$ & 775.2 & & \\
\hline-32.20 & 791.4 & & \\
\hline-30.54 & 802.9 & & \\
\hline-28.88 & 818.5 & & \\
\hline-27.21 & 823.6 & & \\
\hline
\end{tabular}


Table 2. Parameters of the Lennard-Jones and Coulomb Interactions of the Potential Models. $\sigma, \varepsilon$ and $q$ Stand for the Lennard-Jones Distance and Energy Parameters and for the Fractional Charges, Respectively.

\begin{tabular}{ccccc}
\hline \hline Molecule & Site & $\sigma / \AA$ & $\varepsilon / \mathrm{kJ} \mathrm{mol}^{-1}$ & $q / \mathrm{e}$ \\
\hline \multirow{3}{*}{ methylamine $^{\mathrm{a}}$} & $\mathrm{CH}_{3}$ & 3.80 & 0.711 & 0.25 \\
& $\mathrm{~N}$ & 3.30 & 0.711 & -1.05 \\
& $\mathrm{H}$ & - & - & 0.40 \\
& & & & \\
water $^{\mathrm{b}}$ & $\mathrm{O}$ & 3.12 & 0.670 & 0 \\
& $\mathrm{H}$ & - & - & 0.241 \\
& $\mathrm{~L}^{\mathrm{c}}$ & - & - & -0.241 \\
\hline \hline
\end{tabular}

$\overline{{ }^{\mathrm{a}} \text { Ref. } 22} \overline{{ }^{\mathrm{b}} \text { TIP5P model, }} \overline{\text { Ref. } 45} \overline{{ }^{\mathrm{c}} \text { Non-atomic interaction site }}$

Table 3. Bond Lengths and Bond Angles of the Potential Models

\begin{tabular}{ccccc}
\hline \hline molecule & bond & bond length $(\AA)$ & angle & bond angle (deg) \\
\hline \multirow{3}{*}{ methylamine } & $\mathrm{CH}_{3}-\mathrm{N}$ & 1.448 & & \\
& $\mathrm{~N}-\mathrm{H}$ & 1.010 & & \\
& & & $\mathrm{CH}_{3}-\mathrm{N}-\mathrm{H}$ & 109.5 \\
& & & $\mathrm{H}-\mathrm{N}-\mathrm{H}$ & 106.4 \\
& & & & \\
water & $\mathrm{O}-\mathrm{H}$ & 0.957 & & \\
& $\mathrm{O}-\mathrm{L}$ & 0.700 & $\mathrm{H}-\mathrm{O}-\mathrm{H}$ & 104.50 \\
& & & L-O-L & 110.70 \\
\hline \hline
\end{tabular}




\section{Figure Legend}

Figure 1. Adsoprtion isotherm of methylamine (mean number of the adsorbed $\mathrm{CH}_{3} \mathrm{NH}_{2}$ molecules as a function of their chemical potential) at the surface of ice. The line connecting the points only serves as a guide to the eye. The inset depicts the same isotherm in the more conventional $\Gamma$ vs. $p_{\text {rel }}$ form (black circles) up to the point of condensation and the Langmuir isotherm fitted to these data up to a $p_{\text {rel }}$ value of 0.75 (red solid line). The innermost inset shows the turning region of this isotherm on a magnified scale. The arrows mark the systems used for detailed analyses.

Figure 2. Instantaneous equilibrium snapshots of the surface part of systems 1-5 (from left to right), both in top and side views (top and bottom rows, respectively). $\mathrm{CH}_{3}$ groups, $\mathrm{O}, \mathrm{N}$, and $\mathrm{H}$ atoms are marked by black, red, blue, and white colours, respectively; water hydrogens are omitted from the picture.

Figure 3. Number density profile of methylamine along the $X$ (surface normal) axis of the basic box, as obtained in systems 1-5 (red solid line, green dashed line, blue dash-dotted line, orange dash-dot-dotted line, and magenta circles, respectively) The water number density profile corresponding to the outmost layer of ice is also shown (dashed black line). The boundary of the first molecular layer is marked by the dotted vertical line. The water number density profile in the entire ice phase is shown by the inset as obtained in system 1.

Figure 4. Definition of the local Cartesian frame fixed to the individual methylamine molecules, used in the orientational analysis, and of the polar angles $\vartheta$ and $\phi$ of the surface normal vector, $\underline{X}$, pointing away from the ice phase. Colour coding is the same as in Figure 2 . 
Figure 5. Orientational maps of the first layer methylamine molecules as obtained in systems 1, 2, 3 and 5 (from left to right). Lighter colours correspond to higher probabilities. The methylamine orientations corresponding to the various peaks of the orientational maps are also shown. Colour coding is the same as in Figure 2. $\underline{X}$ is the surface normal vector, directed to point from the ice to the methylamine phase.

Figure 6. (a) Hydrogen bonding arrangements of first layer methylamine and surface water molecules, in which all molecules are aligned in one of their preferred surface orientations. The dashed black lines are schematic representations of the hydrogen bonds. (b) Relative arrangements of two neighboring first layer methylamine molecules both aligned in one of their preferred surface orientations, corresponding to favorable dipole-dipole arrangements. The arrows are schematic representations of the molecular dipole vectors Colour coding is the same as in Figure 2. $\underline{X}$ is the surface normal vector, directed to point from the ice to the methylamine phase.

Figure 7. Distribution of the total binding energy of a methylamine molecule in the first adlayer at the ice surface (bottom) and of its contributions due to the interaction with the other methylamine molecules (middle) and with the water molecules of the ice phase (top), as obtained in systems 1, 2, 3, and 5 (red solid lines, green dashed lines, blue dash-dotted lines, and magenta circles, respectively). 
Figure 1

Szentirmai et al.

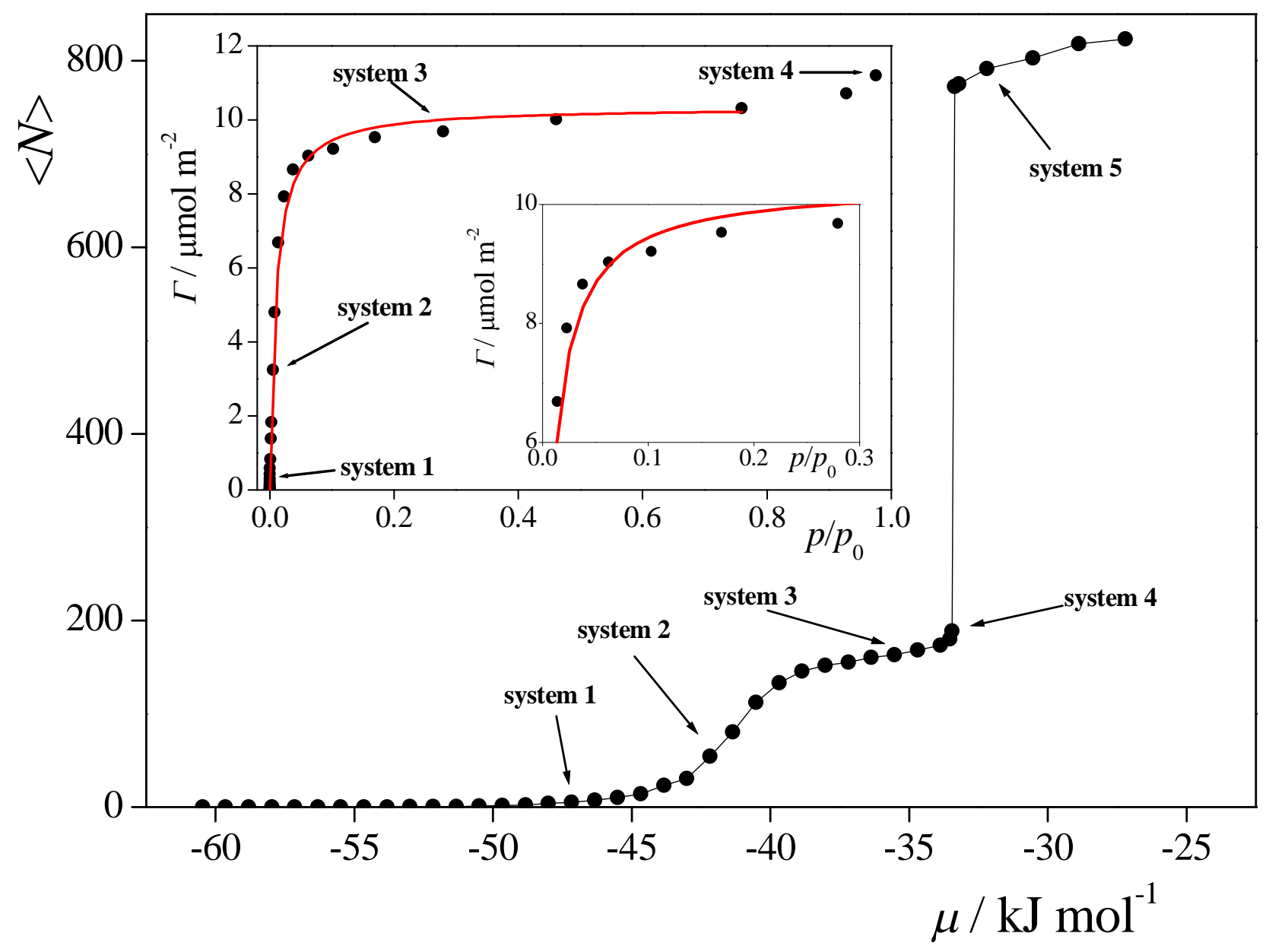


Figure 2

Szentirmai et al.
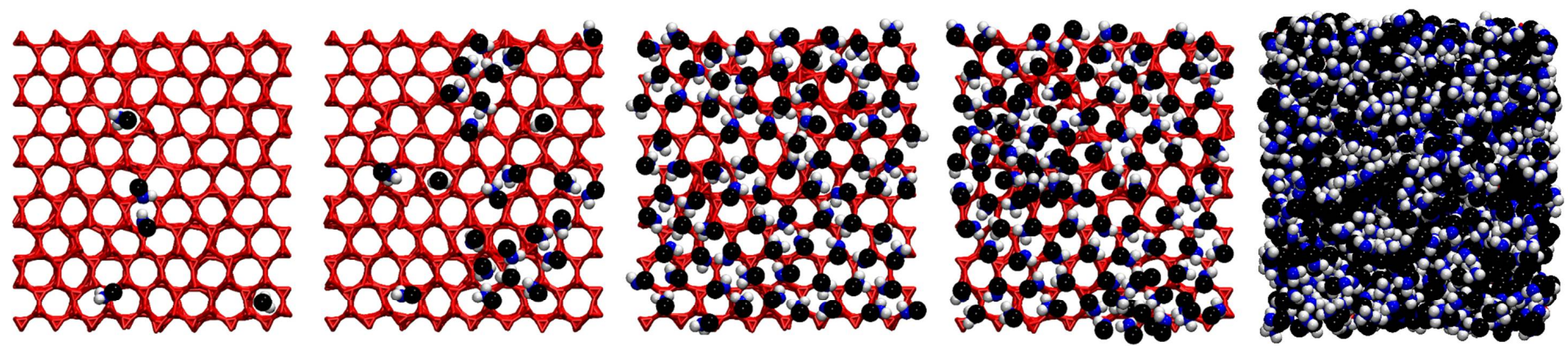

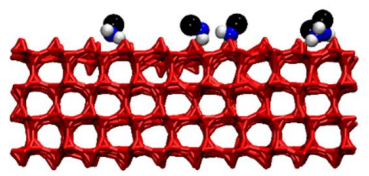
system 1

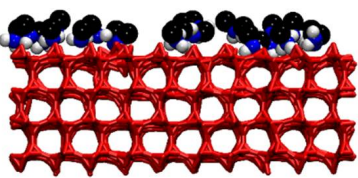

system 2

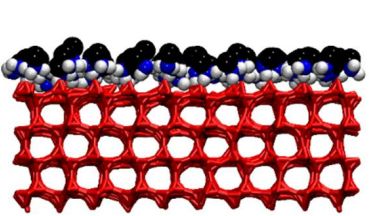

system 3

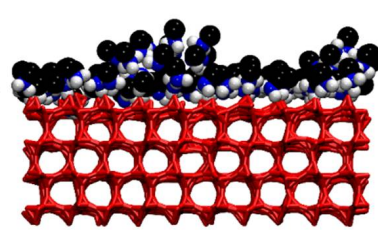

system 4

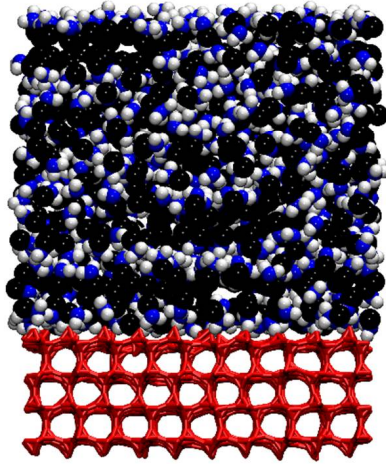

system 5 
Figure 3

Szentirmai et al.

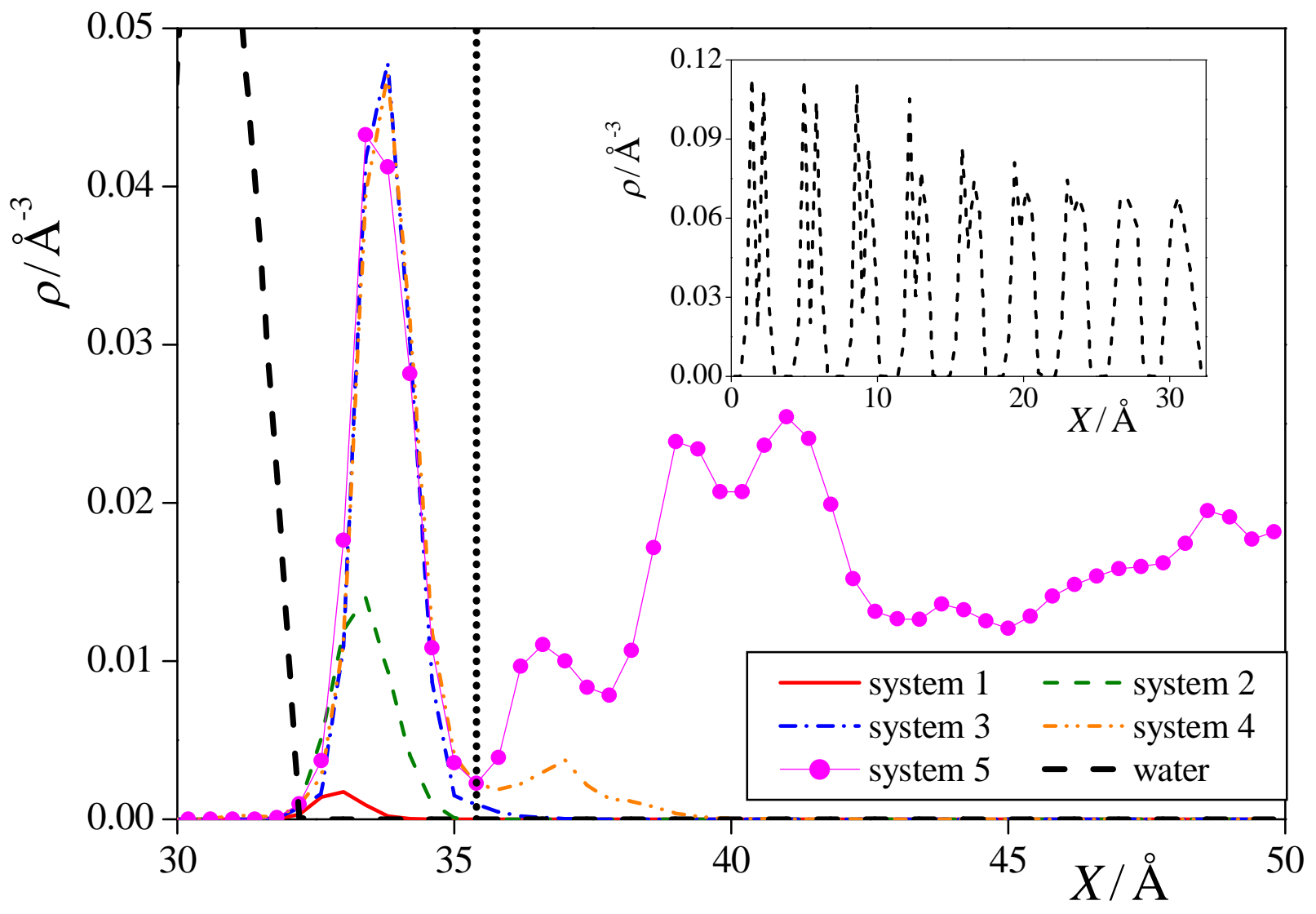


Figure 4

Szentirmai et al.

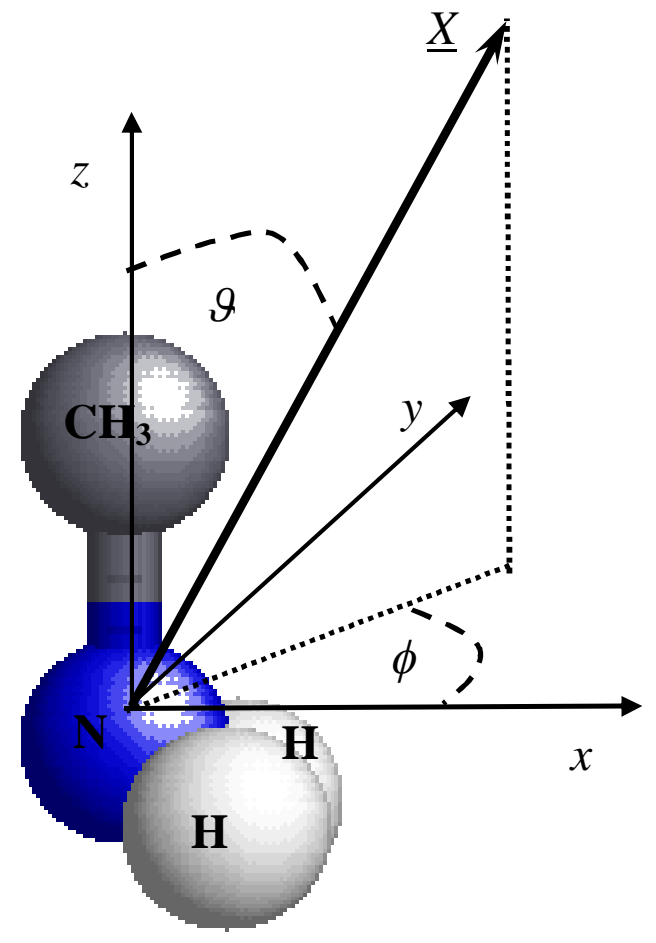


Figure 5

Szentirmai et al.

system 1
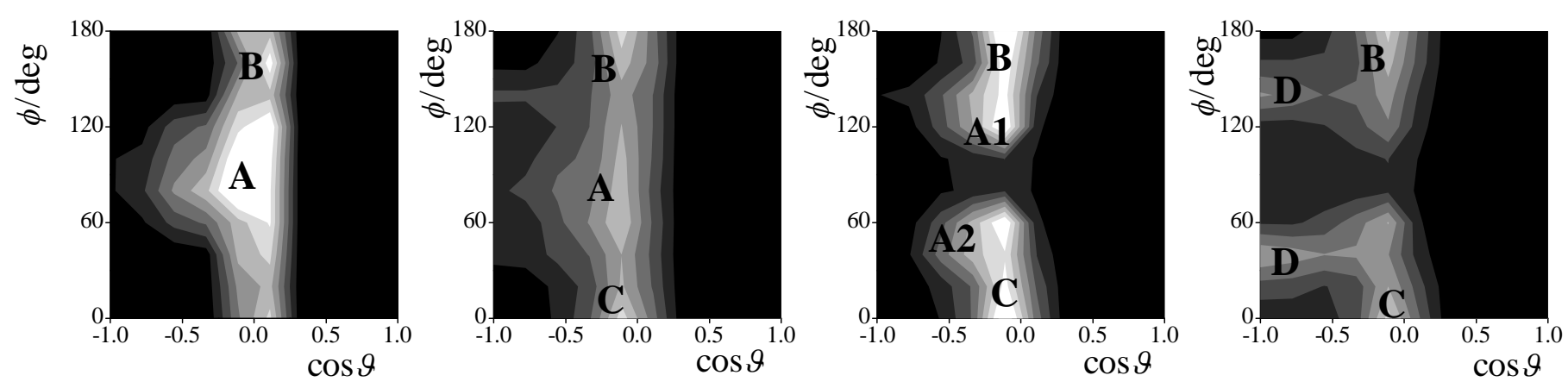

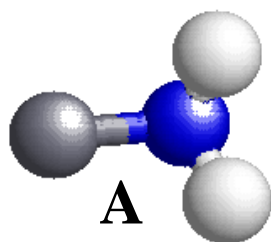

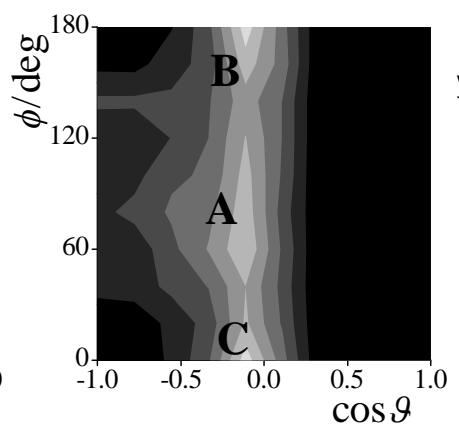

system 2
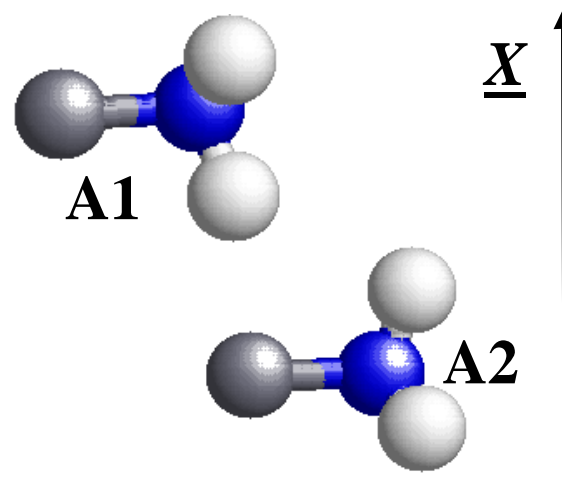

system 3

system 5
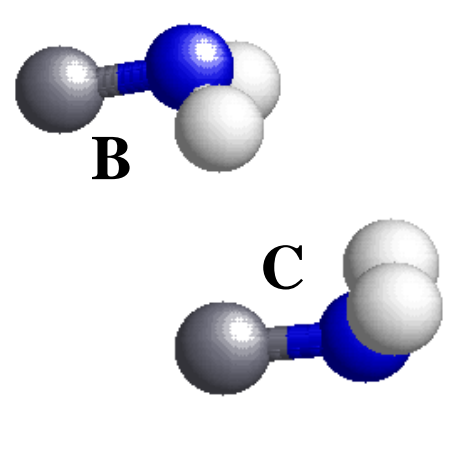
Figure 6.a

Szentirmai et al.

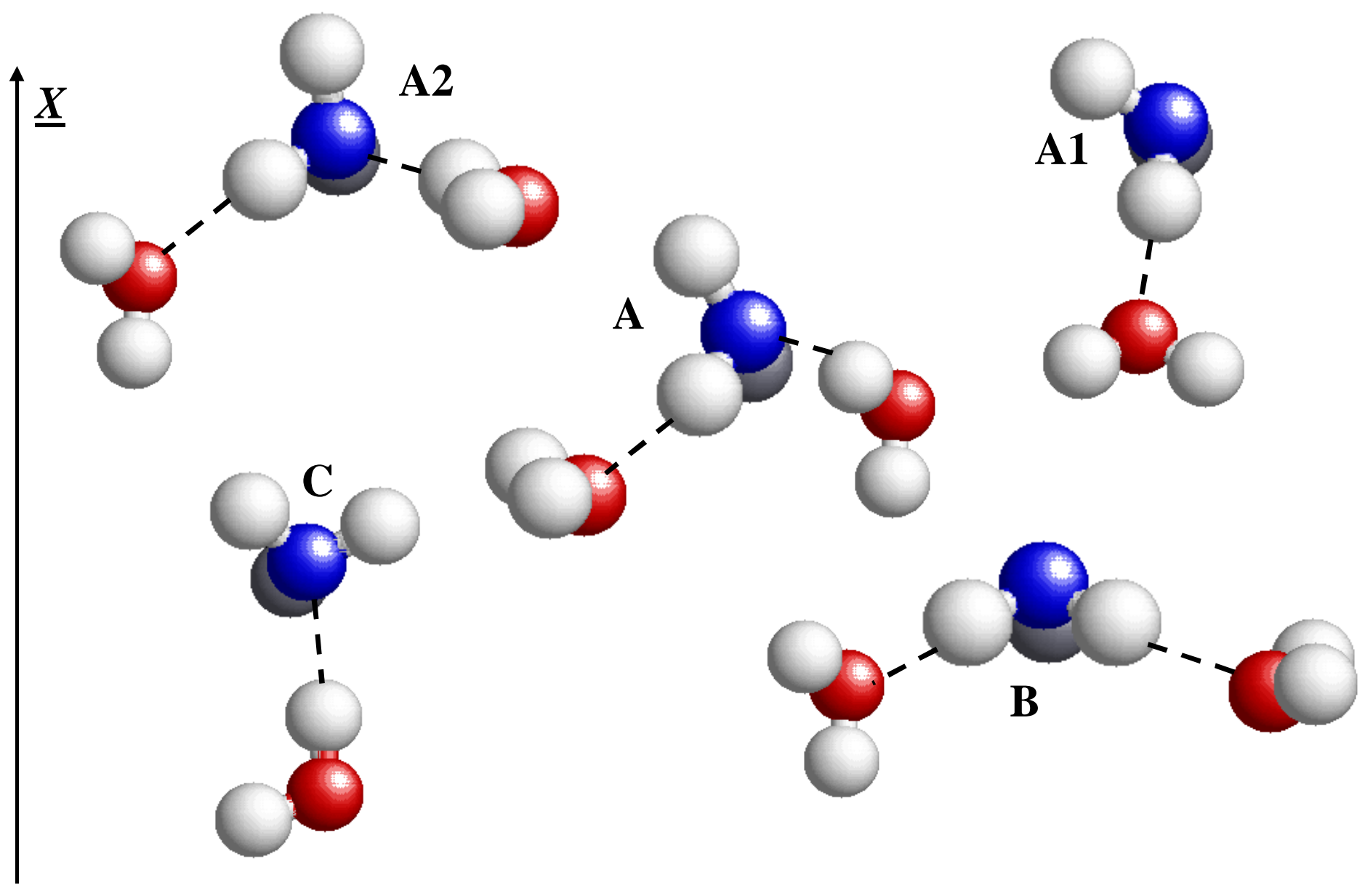


Figure 6.b

Szentirmai et al.
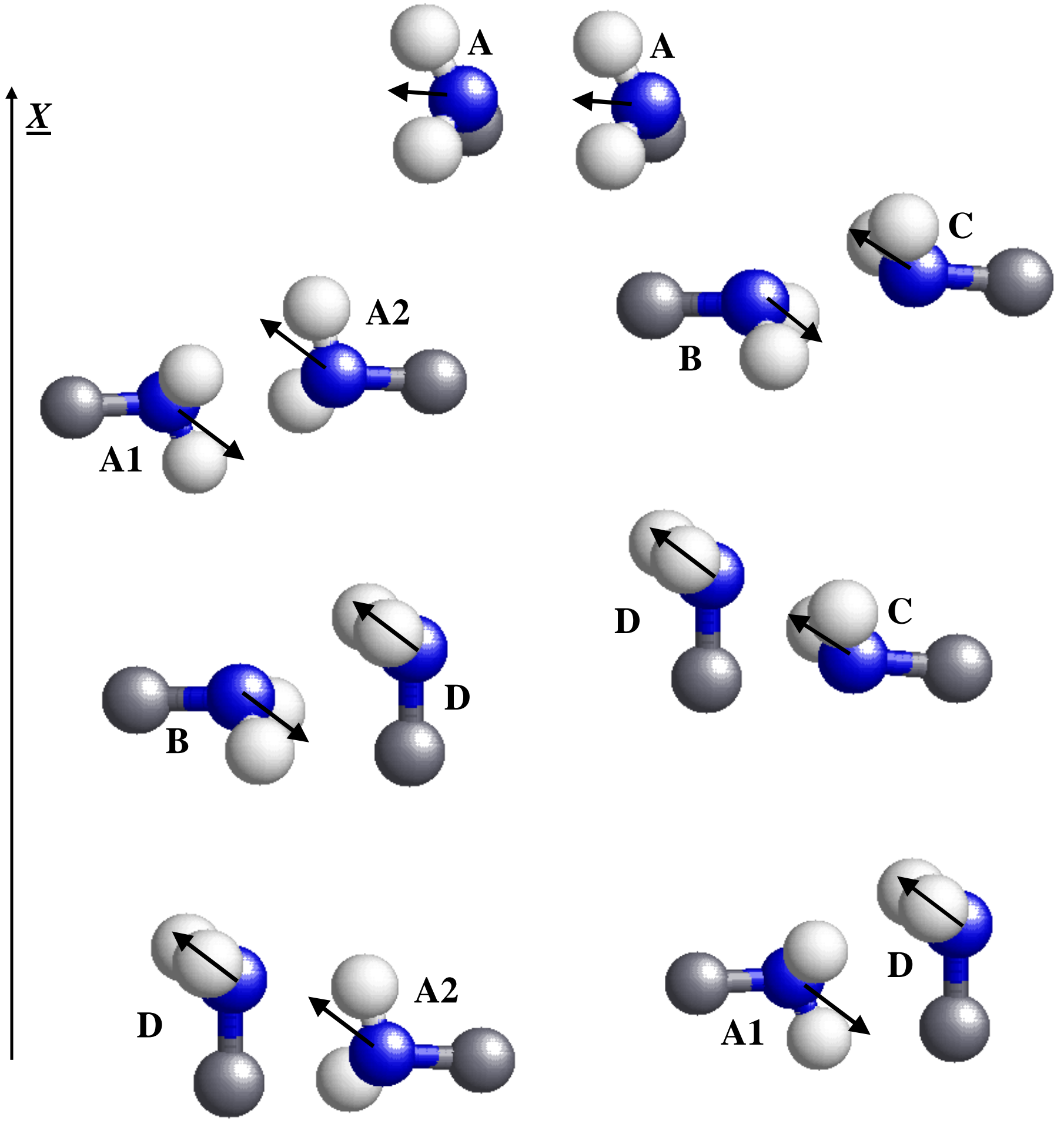
Figure 7

Szentirmai et al.

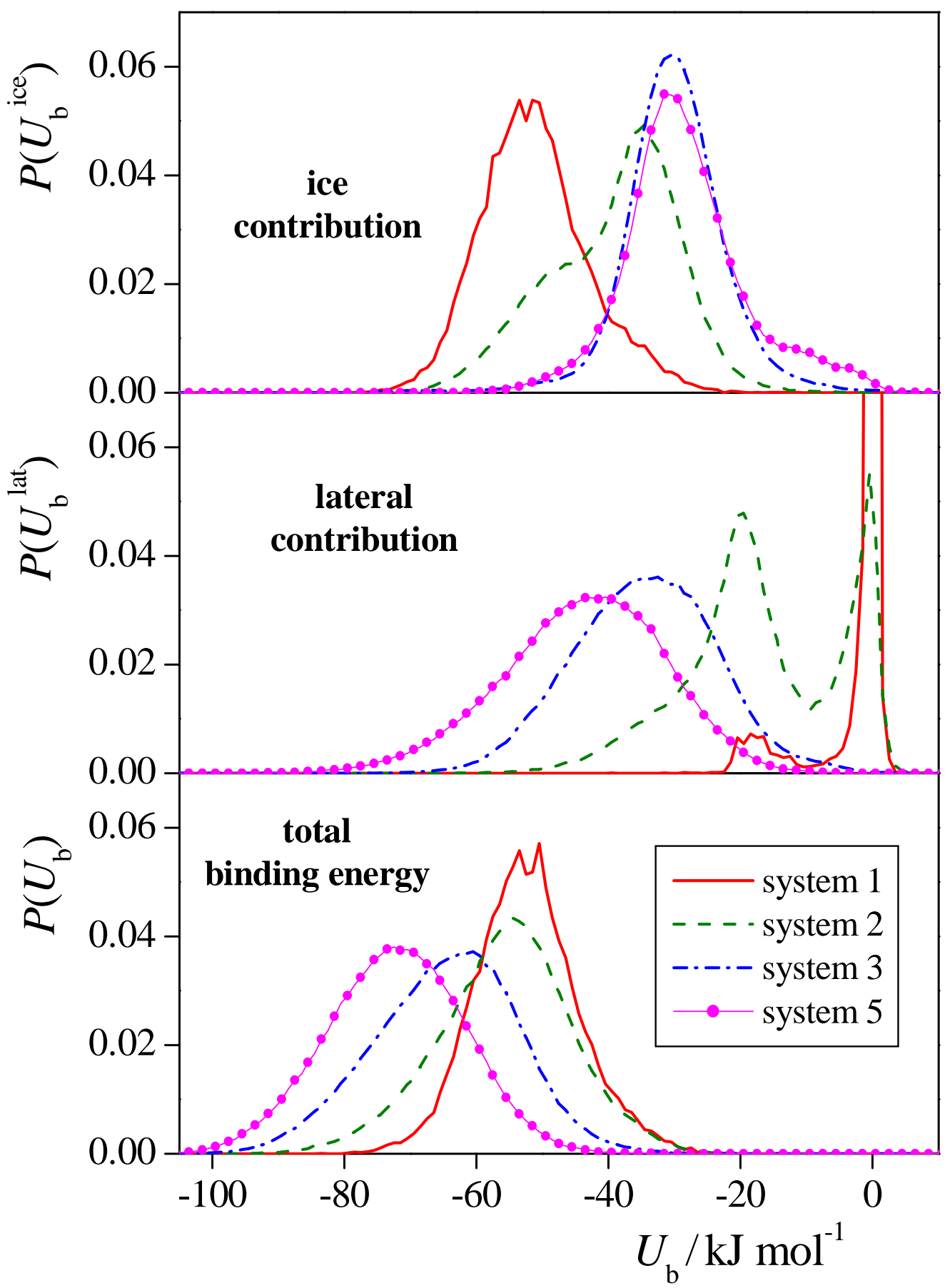


Table of Contents Graphics:

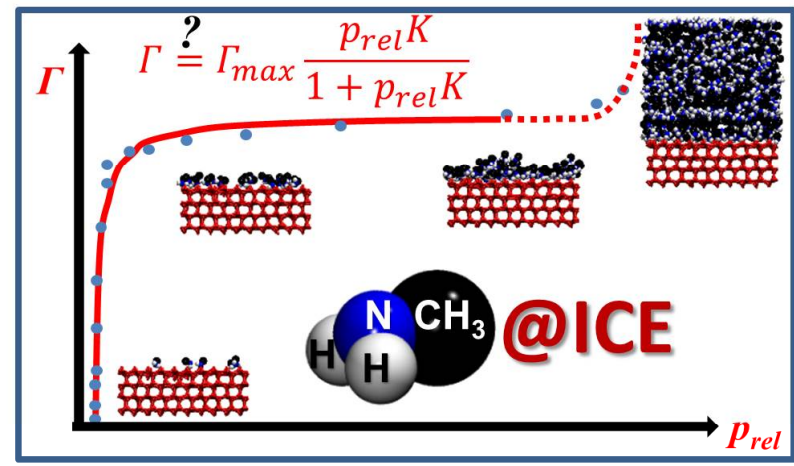

TOC 